\title{
Probabilistic and Interval Analyses Contrasted in Impact Buckling of a Clamped Column
}

\author{
Isaac Elishakoff \\ Professor, \\ Fellow of ASME \\ Florida Atlantic University \\ Boca Raton, \\ Florida, 33431 \\ elsihako@fau.edu \\ David Moens \\ Professor \\ Dept. of Applied Engineering \\ Lessius Mechelen, Campus De Nayer \\ B2860 St-Katelijne-Waver \\ Associated Professor \\ Dept. of Mechanical Engineering \\ KU Leuven \\ B3001 Heverlee \\ Belgium \\ david.moens@mech.kuleuven.be
}

Wim Verhaeghe

PhD. Student,

ASME Student Member

Dept. of Ocean and Mechanical Engineering Dept. of Mechanical Engineering

KU Leuven

B3001 Heverlee

Belgium

wim.verhaeghe@mech.kuleuven.be

In this study we contrast two competing methodologies for the impact buckling of a column that is clamped at its both ends. The initial imperfection is postulated to be coconfigurational with the fundamental mode shape of the column without the axial loading. Solution is also furnished for the case when the initial imperfection is proportional to the "cosinusoidal polynomial" by Filonenko-Borodich. Probabilistic and interval analyses are conducted for each case; these are contrasted on some representative numerical data.

\section{Introduction}

The study of the impact buckling of columns with simply supported boundary conditions and uncertain initial imperfections has been delt with by Lindberg [1], Elishakoff $[2,3]$ and Ben-Haim and Elishakoff [4] and other authors.

Lindberg [1] represented the initial imperfections by a Fourier series in terms of the classical buckling modes. The Fourier coefficients were assumed to be normally distributed random variables with zero mean, and with variance proportional to the power spectral density of the initial imperfection. The latter was assumed to be a band-limited white noise. The expectation of the wavelength, measured between the alternate zero crossings, was evaluated (with the assumption of the homogeneity of random imperfections), and wavelength histrograms were plotted on the basis of 65 computations.

Elishakoff $[2,3]$ treated the uncertain initial imperfections probabilistically via a single [2] or multiple [3] random variables and a corresponding number of deterministic functions satisfying boundary conditions. The safe range of the structure was identified with the absolute value of the total displacement being less than some prescribed value. The difference between [1], on one hand, and [2,3] on the other, is that in contrast to Ref. [1], Refs. [2,3] deal with structural reliability. The probability distribution function of the first excursion time is found in closed form for the three cases of axial load, which is less than, equal to, and greater than the corresponding classical buckling load of a perfect structure [2]. In Ref. [3] the Monte Carlo method is applied to the problem of impact buckling of an elastic bar with finite number of jointly normally distributed initial imperfection coefficients with given mean and autocorrelation functions. For each realization the buckling time is obtained numerically, and the results are used in constructing the empirical reliability function.

The above studies provided a fertile ground for BenHaim and Elishakoff [4] to treat the uncertain initial imperfections by a non-probabilistic, convex model. The calcula- 
tion of the following bounds is carried out: maximum deflection, duration above a threshold and maximum integral displacement. Closed-form solutions were obtained for these bounds. Latest works in this direction are by [5-7]. Lindberg comments on the comparison of the convex and probabilistic model in [8]. It is shown that a clear relationship can be derived between the standard deviation and the maximum displacement in the probabilistic and the convex analysis, respectively.

The effect of boundary conditions was not investigated in above studies. It is the aim of current paper to close this gap. In the present study the problem of impact buckling of a clamped column with uncertain initial imperfections is treated by both convex modelling and probabilistic analysis. Furthermore, the effect of using an approximative solution versus exact solution is studied. To this effect, we utilize on the one hand, the exact mode shapes for a column, clamped at its ends. On the other hand, we utilize so called "cosinusoidal polynomials" by Filonenko-Borodich [9] as the set of comparison functions within the Bubnov-Galerkin methodology.

\section{Governing equation}

Following [10], we disregard axial wave propagation and assume uniform compression throughout the bar, whose motion obeys the following differential equation:

$$
E I \frac{\partial^{4} y}{\partial x^{4}}+P \frac{\partial^{2} y}{\partial x^{2}}+\rho A \frac{\partial^{2} y}{\partial t^{2}}=-P \frac{\partial^{2} \bar{y}}{\partial x^{2}}
$$

Here $x$ is the axial coordinate, $t$ is the time, $\bar{y}(x)$ is the initial imperfection (a small perturbation to the initial shape of the unstressed bar), $y(x, t)$ is the additional transverse deflection measured from $\bar{y}(x), E$ is Young's modulus, $I$ is moment of inertia, $\rho$ is mass density, $A$ is the cross-sectional area, $P$ is the applied axial load, $E I$ and $\rho A$ are taken as constant.

For the clamped-clamped beam, the differential equation (1) is supplemented by the boundary conditions

$$
y(x, t)=0, \quad \frac{\partial y(x, t)}{\partial x}=0 ; \quad \text { at } x=0, l,
$$

and the initial conditions

$$
y(x, t)=0, \quad \frac{\partial y(x, t)}{\partial t}=0 ; \quad \text { at } t=0
$$

The excitation constitutes a compressive step-loading: The constant load $P$ is applied at time $t=0$ and kept indefinitely.

\section{Solution of the deterministic problem}

For the deterministic solution of the problem we introduce following nondimensional quantities

$$
\begin{gathered}
\xi=\frac{x}{l}, \quad \tau=\omega_{1} t, \quad \alpha=\frac{P}{P_{C L}}, \\
u(\xi, \tau)=\frac{y(x, t)}{\Delta}, \quad \bar{u}(\xi)=\frac{\bar{y}(x)}{\Delta}
\end{gathered}
$$

where

$$
P_{C L}=\frac{4 \pi^{2} E I}{l^{2}}, \quad \omega_{1}=\gamma\left(\frac{\pi}{l}\right)^{2} \sqrt{\frac{E I}{\rho A}}, \quad \Delta=\sqrt{\frac{I}{A}}
$$

$P_{C L}$ being the classical buckling load of a perfect bar clamped at both ends, $\omega_{1}$, its fundamental natural frequency in the absence of axial compression, $\Delta$, its radius of gyration, $\alpha$, the nondimensional applied force, $u(\xi, \tau)$ and $\bar{u}(\xi)$ the nondimensional additional and initial displacements, respectively, and $\gamma=4.73^{2} / \pi^{2}=2.27$ according to Ref. [11].

Equation (1) becomes

$$
\frac{\partial^{4} u}{\partial \xi^{4}}+4 \pi^{2} \alpha \frac{\partial^{2} u}{\partial \xi^{2}}+\gamma^{2} \pi^{4} \frac{\partial^{2} u}{\partial \tau^{2}}=-4 \pi^{2} \alpha \frac{\partial^{2} \bar{u}}{\partial \xi^{2}}
$$

\subsection{Initial imperfection that is co-configurational with the exact mode shape}

For the solution method using the exact mode shape, the shape of the imperfection is taken as follows

$$
\bar{y}(x)=H_{0} P_{0}\left(\frac{x}{l}\right)
$$

where

$$
\begin{aligned}
P_{0}\left(\frac{x}{l}\right)= & \cos \left(\sqrt{\gamma} \pi \frac{x}{l}\right)-\cosh \left(\sqrt{\gamma} \pi \frac{x}{l}\right)- \\
& \frac{\cos (\sqrt{\gamma} \pi)-\cosh (\sqrt{\gamma} \pi)}{\sin (\sqrt{\gamma} \pi)-\sinh (\sqrt{\gamma} \pi)}\left[\sin \left(\sqrt{\gamma} \pi \frac{x}{l}\right)-\sinh \left(\sqrt{\gamma} \pi \frac{x}{l}\right)\right]
\end{aligned}
$$

which constitutes the exact mode shape of the column without axial loading [11]. After nondimensionalizing, Eq. (7) becomes

$$
\bar{u}(\xi)=A_{0} P_{0}(\xi)
$$

with $A_{0}=H_{0} / \Delta$. The boundary conditions which follow from substituting (9) in (6) are satisfied by setting:

$$
u(\xi, \tau)=G_{0}(\tau) P_{0}(\xi)
$$


For $P_{0}(\xi)$ the following relation holds:

$$
\frac{d^{4} P_{0}(\xi)}{d \xi^{4}}=\gamma^{2} \pi^{4} P_{0}(\xi)
$$

Combining Eqs. (6),(10) and (11) leads to

$$
\begin{aligned}
\gamma^{2} \pi^{4} P_{0}(\xi) G_{0}(\tau)+ & 4 \pi^{2} \alpha \frac{\partial^{2} P_{0}(\xi)}{\partial \xi^{2}} G_{0}(\tau)+\gamma^{2} \pi^{4} P_{0}(\xi) \frac{\partial^{2} G_{0}(\tau)}{\partial \tau^{2}} \\
& =-4 \pi^{2} \alpha A_{0} \frac{\partial^{2} P_{0}(\xi)}{\partial \xi^{2}}
\end{aligned}
$$

Because of following relations,

$$
\begin{array}{r}
\int_{0}^{1} P_{0}(\xi) P_{0}(\xi) d \xi=1, \\
\int_{0}^{1} P_{0}(\xi) \frac{\partial^{2} P_{0}(\xi)}{\partial \xi^{2}}=-\kappa \frac{\pi^{2}}{4}, \quad \kappa=4.99
\end{array}
$$

the multiplication of (12) with $P_{0}(\xi)$ and subsequent integration over $\xi=0 \ldots 1$ leads to following ordinary differential equation for $G_{0}(\tau)$

$$
\frac{d^{2} G_{0}(\tau)}{d \tau^{2}}+G_{0}(\tau)\left(1-\frac{\kappa}{\gamma^{2}} \alpha\right)=\frac{\kappa}{\gamma^{2}} \alpha A_{0}
$$

The solution of Eq. (14) reads $G_{0}(\tau)=A_{0} \psi_{0}(\tau)$ with

$$
\psi_{0}(\tau)= \begin{cases}\frac{\kappa \alpha}{\kappa \alpha-\gamma^{2}}\left[\cos \left(\tau \sqrt{1-\frac{\kappa}{\gamma^{2}} \alpha}\right)-1\right] & \text { if } \alpha<\frac{\gamma^{2}}{\kappa} \\ \frac{\tau^{2}}{2} & \text { if } \alpha=\frac{\gamma^{2}}{\kappa} \\ \frac{\kappa \alpha}{\kappa \alpha-\gamma^{2}}\left[\cosh \left(\tau \sqrt{\frac{\kappa}{\gamma^{2}} \alpha-1}\right)-1\right] & \text { if } \alpha>\frac{\gamma^{2}}{\kappa}\end{cases}
$$

The total displacement is thus described by:

$$
v(\xi, \tau)=u(\xi, \tau)+\bar{u}(\xi)=A_{0}\left[1+\psi_{0}(\tau)\right] P_{0}(\xi)
$$

Usually the problems are solved via approximate techniques due to possible inhomogeneity or other deviations from uniformity. In order to model the effect of approximate techniques, we provide hereinafter the solution via the Bubnov-Galerkin method utilizing, as a comparison function, so called Filonenko-Borodich "cosinusoidal polynomials".

\subsection{Solution using one term of Filonenko-Borodich se- ries}

In the approximate solution, the shape of the imperfection is taken as follows

$$
\bar{y}^{*}(x)=H_{0}^{*} P_{0}^{*}\left(\frac{x}{l}\right)
$$

where

$$
P_{0}^{*}\left(\frac{x}{l}\right)=1-\cos \left(2 \pi \frac{x}{l}\right)
$$

is the first "cosinusoidal polynomial" by Filonenko-Borodich [9]. Some mathematical properties of these functions were proved by Vilenkin [12]. After nondimensionalizing, Eq. (17) becomes

$$
\bar{u}^{*}(\xi)=A_{0}^{*}[1-\cos (2 \pi \xi)], \quad A_{0}^{*}=\frac{H_{0}^{*}}{\Delta}
$$

The boundary conditions which follow from substituting (19) in (6) are satisfied by setting:

$$
u^{*}(\xi, \tau)=G_{0}^{*}(\tau) P_{0}^{*}(\xi)
$$

The error of this solution equals

$$
\begin{aligned}
\varepsilon(\xi, \tau)= & G_{0}^{*}(\tau) \frac{d^{4} P_{0}^{*}(\xi)}{d \xi^{4}}+4 \pi^{2} \alpha G_{0}^{*}(\tau) \frac{d^{2} P_{0}^{*}(\xi)}{d \xi^{2}} \\
& +\gamma^{2} \pi^{4} P_{0}^{*}(\xi) \frac{\partial^{2} G_{0}^{*}(\tau)}{\partial \tau^{2}}+4 \pi^{2} \alpha A_{0}^{*} \frac{d^{2} P_{0}^{*}(\xi)}{d \xi^{2}}
\end{aligned}
$$

The method of Bubnov-Galerkin stipulates the above error $\varepsilon(\xi, \tau)$ to be orthogonal to $P_{0}^{*}(\xi)$ :

$$
\int_{0}^{1} \varepsilon(\xi, \tau) P_{0}^{*}(\xi) d \xi=0
$$

Because of the orthogonality properties of the function $P_{0}^{*}(\xi)$ and its derivatives $d^{2} P_{0}^{*}(\xi) / d \xi^{2}$ and $d^{4} P_{0}^{*}(\xi) / d \xi^{4}$ :

$$
\begin{array}{r}
\int_{0}^{1} P_{0}^{*}(\xi) P_{0}^{*}(\xi) d \xi=\frac{3}{2}, \quad \int_{0}^{1} \frac{d^{2} P_{0}^{*}(\xi)}{d \xi^{2}} P_{0}^{*}(\xi) d \xi=-2 \pi^{2} \\
\int_{0}^{1} \frac{d^{4} P_{0}^{*}(\xi)}{d \xi^{4}} P_{0}^{*}(\xi)=8 \pi^{4}
\end{array}
$$

following relation is obtained:

$$
\frac{3}{16} \gamma^{2} \frac{d^{2} G_{0}^{*}(\tau)}{d \tau^{2}}+G_{0}^{*}(\tau)(1-\alpha)=\alpha A_{0}^{*}
$$

The solution of equation (24) reads $G_{0}^{*}(\tau)=A_{0}^{*} \psi_{0}^{*}(\tau)$ with

$$
\psi_{0}^{*}(\tau)= \begin{cases}\frac{\alpha}{\alpha-1}\left[\cos \left(\frac{4 \tau}{\sqrt{3} \gamma} \sqrt{1-\alpha}\right)-1\right] & \text { if } \alpha<1 \\ \frac{16}{3 \gamma^{2}} \frac{\tau^{2}}{2} & \text { if } \alpha=1 \\ \frac{\alpha}{\alpha-1}\left[\cosh \left(\frac{4 \tau}{\sqrt{3} \gamma} \sqrt{\alpha-1}\right)-1\right] & \text { if } \alpha>1\end{cases}
$$


The total displacement is thus described by:

$$
v(\xi, \tau)=u(\xi, \tau)+\bar{u}(\xi)=A_{0}^{*}\left[1+\psi_{0}^{*}(\tau)\right] P_{0}^{*}(\xi)
$$

Hereinafter, the amplitude $A_{0}$ or $A_{0}^{*}$ will be either treated as an interval variable or as a random variable. This is done with an express goal of comparing these two philosophically competing methodologies. Now we are interested to find extremal responses when initial imperfection parameter is an interval variable.

\section{Interval model for the initial imperfection}

In the $1 D$ case a convex set degenerates to an interval. We represent the uncertainty in the initial imperfection profile so that we allow the coefficient $A_{0}$ and $A_{0}^{*}$ to vary in an interval with lower bound $L, L^{*}$ and upper bound $U, U^{*}$ for the exact mode solution and the Filonenko-Borodich approximation respectively:

$$
A_{0} \in[L U] \quad A_{0}^{*} \in\left[L^{*} U^{*}\right]
$$

To allow a fair comparison of both solution methods, a calibration process for these intervals is needed. For example, based on measurements one can specify the interval for the initial imperfection for a point along the column. For both shapes, the first exact mode and Filonenko-Borodich first term, to represent this interval, the bounds $L, L^{*}$ and $U, U^{*}$ will generally differ from each other. For example, to have the same interval for the initial deflection of the mid-point of the column: $L^{*}=0.794 L$ and $U^{*}=0.794 U$.

Next, the upper bounds on the maximum deflection and the integral displacements are calculated.

\subsection{Maximum Deflection}

A widely accepted criterion for failure belongs to Hoff [13]; it is associated with the maximum deflection: If the instantaneous displacement exceeds a specified threshold, then failure is presumed to result. Because $v(\xi, \tau)$ and equivalently $v^{*}(\xi, \tau)$ are linear functions of the coefficients, and because an interval is the simplest form of a convex set, the maximum of $v$ or $v^{*}$ occurs on the set $C=\{L, U\}$ or $C^{*}=\left\{L^{*}, U^{*}\right\}$ of extreme points of the respective interval. More specifically, the maximum of $\{|L|,|U|\}$ or $\left\{\left|L^{*}\right|,\left|U^{*}\right|\right\}$ will result in the maximum deflection. The maximum for the total displacement of the bar at time $\tau$ and position $\xi$ is

$$
\begin{aligned}
v_{\max }(\xi, \tau) & =\max _{A_{0} \in[L U]} v(\xi, \tau) \\
& =\max \{|L|,|U|\}\left[1+\psi_{0}(\tau)\right] P_{0}(\xi) \\
v_{\text {max }}^{*}(\xi, \tau) & =\max _{A_{0}^{*} \in\left[L^{*} U^{*}\right]} v^{*}(\xi, \tau) \\
& =\max \left\{\left|L^{*}\right|,\left|U^{*}\right|\right\}\left[1+\psi_{0}^{*}(\tau)\right] P_{0}^{*}(\xi)
\end{aligned}
$$

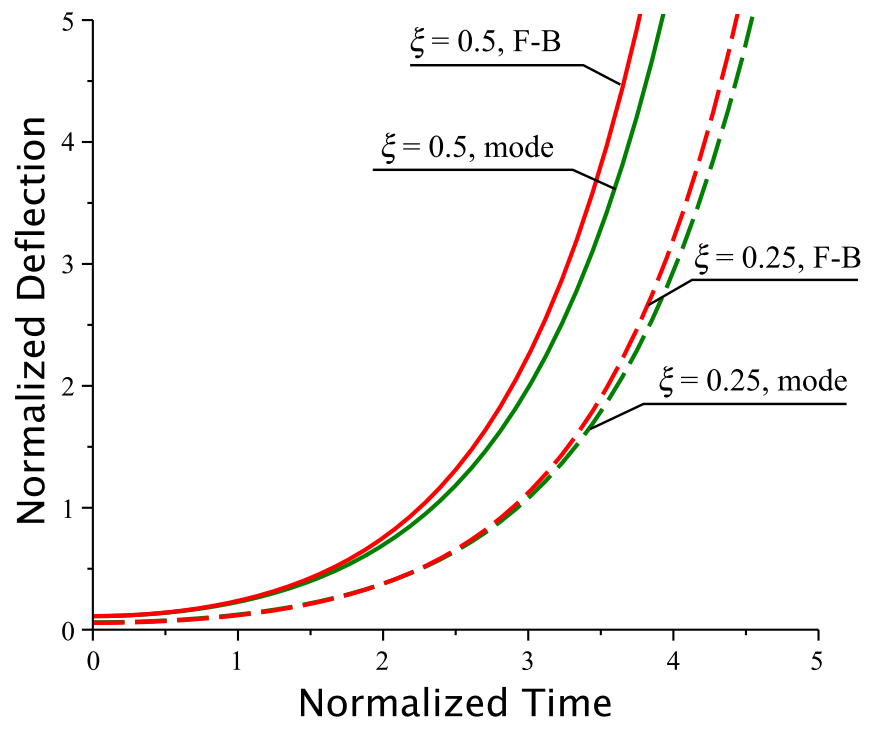

Fig. 1. Maximum normalized deflection versus normalized time at $\xi=0.25$ (dashed lines) and $\xi=0.5$ (solid lines). Load ratio $\alpha=2$. The green lines correspond to the solution with the normal mode; the red lines correspond to the solution with one term Filonenko-Borodich (F-B) approximation

for the solution with the Filonenko-Borodich term and the solution with the normal mode, respectively. Let us consider a numerical example to shed some light on the failure of the clamped-clamped bar. The set of allowed amplitudes is $A_{0} \in$ $[-0.070 .07]$ and $A_{0}^{*} \in[-0.0550 .055]$. The value for $\gamma$ in the expression for the natural frequency $\omega_{1}$ is 2.27 . The value for $\kappa$ appearing in the normal mode solution is 4.99 .

Figure 1 shows the maximum total deflection at two points on the bar axis $(\xi=0.25$ and $\xi=0.5)$ as a function of time. The axial load ratio is set $\alpha=2$. The deflection at $\xi=0.5$ exceeds the deflection at $\xi=0.25$ for all $\tau$ and for both solutions. The normal mode solution appears to give a lower value for the maximum deflection for all shown time instances at both locations $\xi=0.25$ and $\xi=0.5$.

Figure 2 shows the maximum normalized deflection at $\xi=0.5$ as a function of time, for several values of the axial load ratio. The maximum deflection increases sharply as the axial load is increased. The normal mode solution appears to give a lower value for the maximum deflection for all shown time instances and calculated load ratios.

\subsection{Maximum Integral Displacements}

Sometimes researchers are interested in evaluating the integral displacement along the bar at time $\tau$

$$
\delta(\tau)=\int_{0}^{1} \nu(\xi, \tau) d \xi=A_{0} \beta\left(1+\psi_{0}(\tau)\right)
$$

$$
\delta^{*}(\tau)=\int_{0}^{1} v^{*}(\xi, \tau) d \xi=A_{0}^{*}\left(1+\psi_{0}^{*}(\tau)\right)
$$




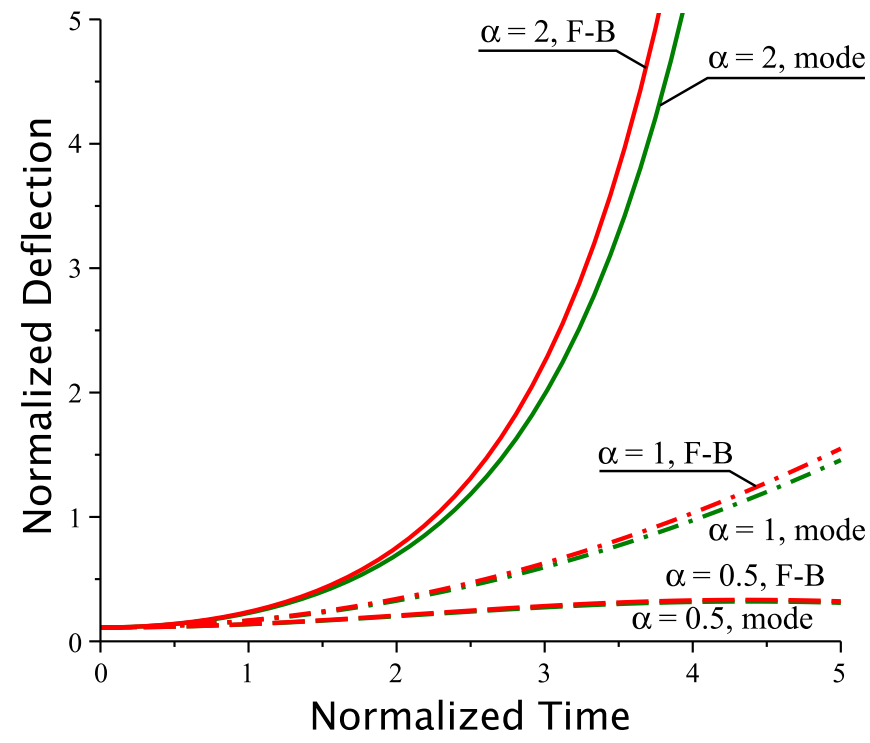

Fig. 2. Maximum normalized deflection versus normalized time at $\xi=0.5$ for various values of the load ratio. The green lines correspond to the solution with the normal mode; the red lines correspond to the solution with one term Filonenko-Borodich (F-B) approximation

since the integral of $P_{0}(\xi)$ and $P_{0}^{*}(\xi)$ over the length of the bar are equal to $\beta \approx 0.831$ and one, respectively.

To calculate the maximum integral deflection for the requirement that $A_{0} \in[L U]$ or $A_{0}^{*} \in\left[L^{*} U^{*}\right]$, the same procedure as in section 4.1 is followed:

$$
\begin{aligned}
& \delta_{\max }(\tau)=U \beta\left[1+\psi_{0}(\tau)\right] \\
& \delta_{\max }^{*}(\tau)=U^{*}\left[1+\psi_{0}^{*}(\tau)\right]
\end{aligned}
$$

Let us consider a numerical example with the same values as above: the set of allowed amplitudes $A_{0} \in[-0.070 .07]$ and $A_{0}^{*} \in[-0.0550 .055]$, the value for $\gamma$ in the expression for the natural frequency $\omega_{1}$ is 2.27 and the value for $\kappa$ appearing in the normal mode solution is 4.99 . Figure 3 shows the maximum span-averaged displacement versus the normalized time, for the load ratio $\alpha=0.5$ and $\alpha=2$ for both solutions. For this measure, the solution with the normal mode and the one term Filonenko-Borodich approximation appear to be very close to each other.

\section{Probabilistic Model for the Initial Imperfection}

Following Ref. [2], let us consider $A_{0}$ and $A_{0}^{*}$ as continuous random variables with probability distribution function $F_{A_{0}}\left(a_{0}\right)$ or $F_{A_{0}^{*}}\left(a_{0}^{*}\right)$. For simplicity, we consider the case of symmetrical bounds for $A_{0}: A_{0} \in[L U]$ with $L=-U$ and equivalently for $A_{0}^{*}$. The failure is identified with the reaching by the absolute value of the total displacements the critical point $c$.

We are interested in the reliability. This means we are interested in the probability $\operatorname{Prob}(\Lambda \geq \lambda)$ of the first excursion

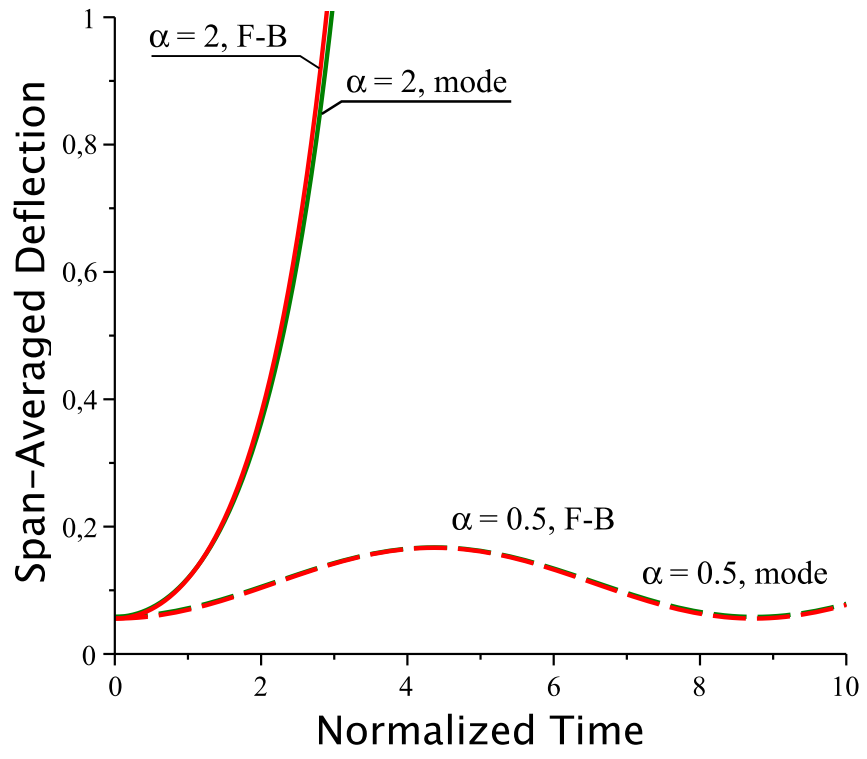

Fig. 3. Maximum integral (span-averaged) deflection versus normalized time for various values of the load ratio. The green lines correspond to the solution with the normal mode; the red lines correspond to the solution with one term Filonenko-Borodich (F-B) approximation

time $\Lambda$ being equal to or greater than the given time $\lambda$. We now proceed to calculate $R(\lambda)$ for three cases, in accordance with equation (26) and $\xi=0.5$ first for the solution with the normal mode and then for the solution with one term of the Filonenko-Borodich approximation.

\subsection{Exact mode shape}

Case 1: $\left(\alpha<\frac{\gamma^{2}}{\kappa}\right)$. In this case buckling is not a certain event. Buckling is possible if

$$
\max _{\tau} v(0.5, \tau)=\left|A_{0}\right| \mu \frac{\gamma^{2}+\kappa \alpha}{\gamma^{2}-\kappa \alpha}, \quad \mu=P_{0}(0.5)
$$

reaches the critical value $c$. Since $\tau=\gamma \pi / \sqrt{\gamma^{2}-\kappa \alpha}$ results in this biggest possible deflection of the mid-point of the bar, the probability that the bar has not buckled is

$$
\begin{aligned}
R(\lambda) & =\operatorname{Prob}\left(\Lambda \geq \frac{\gamma \pi}{\sqrt{\gamma^{2}-\kappa \alpha}}\right)=\operatorname{Prob}\left\{\left|A_{0}\right| \mu \frac{\gamma^{2}+\kappa \alpha}{\gamma^{2}-\kappa \alpha} \leq c\right\} \\
& =F_{A_{0}}\left[\frac{c}{\mu} \frac{\gamma^{2}-\kappa \alpha}{\gamma^{2}+\kappa \alpha}\right]-F_{A_{0}}\left[\frac{-c}{\mu} \frac{\gamma^{2}-\kappa \alpha}{\gamma^{2}+\kappa \alpha}\right]
\end{aligned}
$$

In general, the first-passage time $\lambda$ satisfies the equation

$$
\frac{\mu\left|A_{0}\right|}{\gamma^{2}-\kappa \alpha}\left[\gamma^{2}-\kappa \alpha \cos \left(\lambda \sqrt{1-\frac{\kappa}{\gamma^{2}} \alpha}\right)\right]=c
$$


and for $\lambda \geq 0$ the reliability is calculated as

$$
\begin{aligned}
R(\lambda) & =\operatorname{Prob}(\Lambda \geq \lambda)= \\
\operatorname{Prob}\{ & \left\{\left[A_{0} \geq-c \frac{\gamma^{2}-\kappa \alpha}{\mu} \frac{1}{\gamma^{2}-\kappa \alpha \cos \left(\lambda \sqrt{1-\frac{\kappa}{\gamma^{2}} \alpha}\right)}\right]\right. \\
\cap & {\left.\left[A_{0} \leq c \frac{\gamma^{2}-\kappa \alpha}{\mu} \frac{1}{\gamma^{2}-\kappa \alpha \cos \left(\lambda \sqrt{1-\frac{\kappa}{\gamma^{2}} \alpha}\right)}\right]\right\} } \\
= & {\left[\begin{array}{l}
F_{A_{0}} \\
\left.\left.c \frac{\gamma^{2}-\kappa \alpha}{\mu} \frac{1}{\gamma^{2}-\kappa \alpha \cos \left(\lambda \sqrt{1-\frac{\kappa}{\gamma^{2}} \alpha}\right)}\right]\right\} \\
-F_{A_{0}}\left[-c \frac{\gamma^{2}-\kappa \alpha}{\mu} \frac{\gamma^{2}-\kappa \alpha \cos \left(\lambda \sqrt{1-\frac{\kappa}{\gamma^{2}} \alpha}\right)}{\gamma^{2}}\right]
\end{array}\right.}
\end{aligned}
$$

while for $\lambda<0$, the $\operatorname{Prob}(\Lambda \geq \lambda)=1$. For symmetrically distributed random imperfections, equation (48) becomes

$$
\begin{aligned}
R(\lambda) & =\operatorname{Prob}(\Lambda \geq \lambda) \\
& =1-2 F_{A_{0}}\left[-c \frac{\gamma^{2}-\kappa \alpha}{\mu} \frac{1}{\gamma^{2}-\kappa \alpha \cos \left(\lambda \sqrt{1-\frac{\kappa}{\gamma^{2}} \alpha}\right)}\right]
\end{aligned}
$$

Case 2: $\left(\alpha=\frac{\gamma^{2}}{\kappa}\right)$. In this case, buckling is a certain event. The first excursion time satisfies the equation

$$
\left|A_{0}\right| \mu\left(1+\frac{\lambda^{2}}{2}\right)=c
$$

The reliability $R(\lambda)(\lambda \geq 0)$ is given by

$$
\begin{aligned}
R(\lambda) & =\operatorname{Prob}\left\{\left[A_{0} \geq \frac{-c}{\mu\left(1+\frac{\lambda^{2}}{2}\right)}\right] \cap\left[A_{0} \leq \frac{c}{\mu\left(1+\frac{\lambda^{2}}{2}\right)}\right]\right\} \\
& =F_{A_{0}}\left[\frac{c}{\mu\left(1+\frac{\lambda^{2}}{2}\right)}\right]-F_{A_{0}}\left[\frac{-c}{\mu\left(1+\frac{\lambda^{2}}{2}\right)}\right]
\end{aligned}
$$

whereas for $(\lambda<0)$ the reliability is given by $R(\lambda)=1$. For the symmetrically distributed initial imperfections, we have

$$
F_{\Lambda}(\lambda)=1-2 F_{A_{0}}\left[\frac{-c}{\mu\left(1+\frac{\lambda^{2}}{2}\right)}\right]
$$

Case 3: $\left(\alpha>\frac{\gamma^{2}}{\kappa}\right)$. In this case too, buckling is a certain event. The first excursion time satisfies the equation

$$
\left|A_{0}\right| \frac{\mu}{\kappa \alpha-\gamma^{2}}\left[\kappa \alpha \cosh \left(\lambda \sqrt{\frac{\kappa}{\gamma^{2}} \alpha-1}\right)-\gamma^{2}\right]=c
$$

The reliability $R(\lambda)(\lambda \geq 0)$ is given by

$$
\begin{aligned}
& R(\lambda)= \\
& \operatorname{Prob}\left\{\left[A_{0} \geq-c \frac{\kappa \alpha-\gamma^{2}}{\mu} \frac{1}{\kappa \alpha \cosh \left(\lambda \sqrt{\frac{\kappa}{\gamma^{2}} \alpha-1}\right)-\gamma^{2}}\right]\right. \\
& \left.\cap\left[A_{0} \leq c \frac{\kappa \alpha-\gamma^{2}}{\mu} \frac{1}{\kappa \alpha \cosh \left(\lambda \sqrt{\frac{\kappa}{\gamma^{2}} \alpha-1}\right)-\gamma^{2}}\right]\right\} \\
& =F_{A_{0}}\left[c \frac{\kappa \alpha-\gamma^{2}}{\mu} \frac{1}{\kappa \alpha \cosh \left(\lambda \sqrt{\frac{\kappa}{\gamma^{2}} \alpha-1}\right)-\gamma^{2}}\right] \\
& -F_{A_{0}}\left[-c \frac{\kappa \alpha-\gamma^{2}}{\mu} \frac{1}{\kappa \alpha \cosh \left(\lambda \sqrt{\frac{\kappa}{\gamma^{2}} \alpha-1}\right)-\gamma^{2}}\right]
\end{aligned}
$$

whereas for $(\lambda<0)$ the reliability is given by $R(\lambda)=1$. When the initial imperfections are symmetrically distributed in the positive and negative domains, we obtain following results

$F_{\Lambda}(\lambda)=1-2 F_{A_{0}}\left[-c \frac{\kappa \alpha-\gamma^{2}}{\mu} \frac{1}{\kappa \alpha \cosh \left(\lambda \sqrt{\frac{\kappa}{\gamma^{2}} \alpha-1}\right)-\gamma^{2}}\right]$

\subsection{Reliability within Filonenko-Borodich approxima- tion}

Case 1: $(\alpha<1)$. In this case buckling is not a certain event. Buckling phenomenon is possible if

$$
\max _{\tau} v^{*}(0.5, \tau)=\left|A_{0}^{*}\right| 2 \frac{1+\alpha}{1-\alpha}
$$

reaches the critical value $c$. Since $\tau=\sqrt{3} \gamma \pi / 4 \sqrt{1-\alpha}$ results in this biggest possible deflection of the mid-point of the bar, the probability that the bar has not buckled is

$$
\begin{aligned}
R^{*}(\lambda) & =\operatorname{Prob}\left(\Lambda \geq \frac{\sqrt{3} \gamma \pi}{4 \sqrt{1-\alpha}}\right)=\operatorname{Prob}\left(2\left|A_{0}^{*}\right| \frac{1+\alpha}{1-\alpha} \leq c\right) \\
& =F_{A_{0}^{*}}\left[\frac{c}{2} \frac{(1-\alpha)}{(1+\alpha)}\right]-F_{A_{0}^{*}}\left[-\frac{c}{2} \frac{(1-\alpha)}{(1+\alpha)}\right]
\end{aligned}
$$


In general, the first-passage time $\lambda$ satisfies the equation

$$
\frac{2\left|A_{0}^{*}\right|}{1-\alpha}\left[1-\alpha \cos \left(\frac{4 \lambda}{\sqrt{3} \gamma} \sqrt{1-\alpha}\right)\right]=c
$$

and for $\lambda \geq 0$ the reliability is calculated as

$$
\begin{aligned}
R^{*}(\lambda) & =\operatorname{Prob}(\Lambda \geq \lambda)= \\
& \operatorname{Prob}\left\{\left[A_{0}^{*} \geq-c \frac{1-\alpha}{2} \frac{1}{1-\alpha \cos \left(\frac{4 \lambda}{\sqrt{3} \gamma} \sqrt{1-\alpha}\right)}\right]\right. \\
& \left.\cap\left[A_{0}^{*} \leq c \frac{1-\alpha}{2} \frac{1}{1-\alpha \cos \left(\frac{4 \lambda}{\sqrt{3} \gamma} \sqrt{1-\alpha}\right)}\right]\right\} \\
& =F_{A_{0}^{*}}\left[c \frac{1-\alpha}{2} \frac{1}{1-\alpha \cos \left(\frac{4}{\sqrt{3} \gamma} \sqrt{1-\alpha} \lambda\right)}\right] \\
& -F_{A_{0}^{*}}\left[-c \frac{1-\alpha}{2} \frac{1}{1-\alpha \cos \left(\frac{4 \lambda}{\sqrt{3} \gamma} \sqrt{1-\alpha}\right)}\right]
\end{aligned}
$$

while for $\lambda<0, R^{*}(\lambda)=\operatorname{Prob}(\Lambda \geq \lambda)=1$. For symmetrically distributed random imperfections, equation (48) becomes

$$
\begin{aligned}
R^{*}(\lambda) & =\operatorname{Prob}(\Lambda \geq \lambda) \\
& =1-2 F_{A_{0}^{*}}\left[-c \frac{1-\alpha}{2} \frac{1}{1-\alpha \cos \left(\frac{4 \lambda}{\sqrt{3} \gamma} \sqrt{1-\alpha}\right)}\right]
\end{aligned}
$$

Case 2: $(\alpha=1)$. In this case, buckling is a certain event. The first excursion time satisfies the equation

$$
\left|A_{0}^{*}\right|\left(2+\frac{16}{3 \gamma^{2}} \lambda^{2}\right)=c
$$

The reliability $R^{*}(\lambda)(\lambda \geq 0)$ is given by

$$
\begin{aligned}
R^{*}(\lambda) & =\operatorname{Prob}\left\{\left[A_{0}^{*} \geq \frac{-c}{2+\frac{16}{3 \gamma^{2}} \lambda^{2}}\right] \cap\left[A_{0}^{*} \leq \frac{c}{2+\frac{16}{3 \gamma^{2}} \lambda^{2}}\right]\right\} \\
& =F_{A_{0}^{*}}\left[\frac{c}{2+\frac{16}{3 \gamma^{2}} \lambda^{2}}\right]-F_{A_{0}^{*}}\left[\frac{-c}{2+\frac{16}{3 \gamma^{2}} \lambda^{2}}\right]
\end{aligned}
$$

whereas for $(\lambda<0)$ the reliability is given by $R^{*}(\lambda)=1$. For the symmetrically distributed initial imperfections, we have

$$
R^{*}(\lambda)=1-2 F_{A_{0}^{*}}\left[\frac{-c}{2+\frac{16}{3 \gamma^{2}} \lambda^{2}}\right]
$$

Case 3: $(\alpha>1)$. In this case too, buckling is a certain event. The first excursion time satisfies the equation

$$
\left|A_{0}^{*}\right| \frac{2}{\alpha-1}\left[\alpha \cosh \left(\frac{4 \lambda}{\sqrt{3} \gamma} \sqrt{\alpha-1}\right)-1\right]=c
$$

The reliability $R^{*}(\lambda)(\lambda \geq 0)$ is given by

$$
\begin{aligned}
R^{*}(\lambda) & =\operatorname{Prob}\left\{\left[A_{0}^{*} \geq-c \frac{\alpha-1}{2} \frac{1}{\alpha \cosh \left(\frac{4 \lambda}{\sqrt{3} \gamma} \sqrt{\alpha-1}\right)-1}\right]\right. \\
& \left.\cap\left[A_{0}^{*} \leq c \frac{\alpha-1}{2} \frac{1}{\alpha \cosh \left(\frac{4 \lambda}{\sqrt{3} \gamma} \sqrt{\alpha-1}\right)-1}\right]\right\} \\
& =F_{A_{0}^{*}}\left[c \frac{\alpha-1}{2} \frac{\alpha \cosh \left(\frac{4 \lambda}{\sqrt{3} \gamma} \sqrt{\alpha-1}\right)-1}{\alpha}\right] \\
& -F_{A_{0}^{*}}\left[-c \frac{\alpha-1}{2} \frac{1}{\alpha \cosh \left(\frac{4 \lambda}{\sqrt{3} \gamma} \sqrt{\alpha-1}\right)-1}\right]
\end{aligned}
$$

whereas for $(\lambda<0)$ the reliability is given by $R^{*}(\lambda)=1$. When the initial imperfections are symmetrically distributed in the positive and negative domains, we obtain following result

$$
R^{*}(\lambda)=1-2 F_{A_{0}^{*}}\left[-c \frac{\alpha-1}{2} \frac{1}{-1+\alpha \cosh \left(\frac{4 \lambda}{\sqrt{3} \gamma} \sqrt{\alpha-1}\right)}\right]
$$

\subsection{Numerical examples}

Let us consider a numerical example. Assume that the functions $F_{A_{0}}\left(a_{0}\right)$ and $F_{A_{0}^{*}}\left(a_{0}^{*}\right)$ are given by

$$
\begin{gathered}
F_{A_{0}}\left(a_{0}\right)= \begin{cases}0 & \text { if } A_{0}<L=-0.07 \\
\frac{A_{0}-L}{U-L} & \text { if } L \leq A_{0} \leq U \\
1 & \text { if } A_{0}>U=0.07\end{cases} \\
F_{A_{0}^{*}}\left(a_{0}^{*}\right)= \begin{cases}0 & \text { if } A_{0}^{*}<L^{*}=-0.055 \\
\frac{A_{0}^{*}-L^{*}}{U^{*}-L^{*}} & \text { if } L^{*} \leq A_{0}^{*} \leq U^{*} \\
1 & \text { if } A_{0}^{*}>U^{*}=0.055\end{cases}
\end{gathered}
$$

so that the initial imperfection at the mid point of the beam has the same probability distribution function. Figures 4, 5 


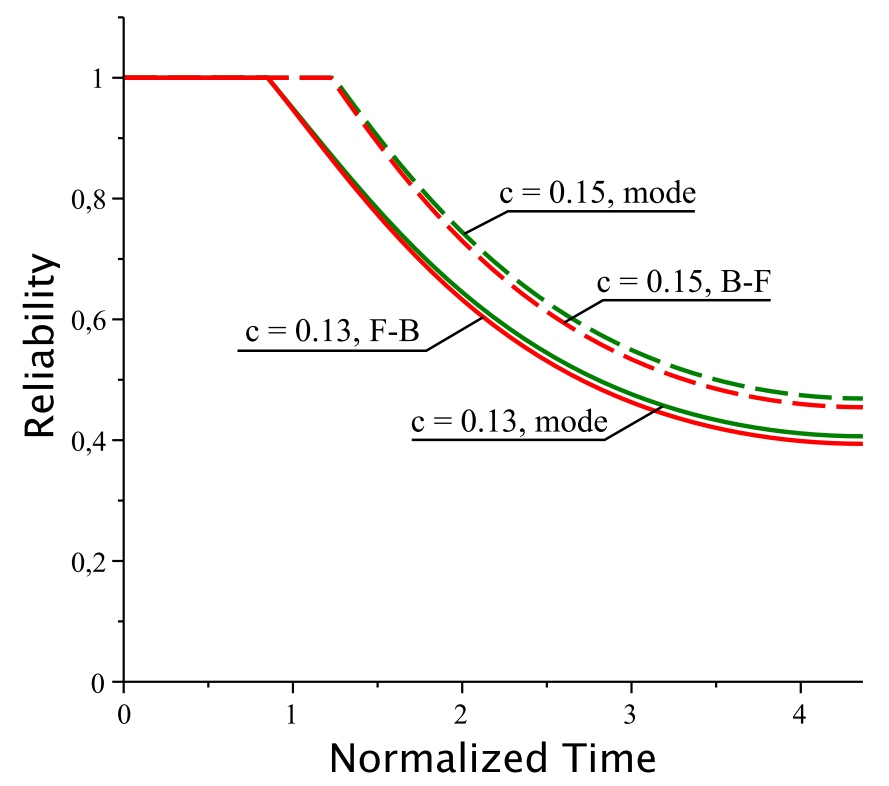

Fig. 4. Reliability as a function of time $(\alpha=0.5)$

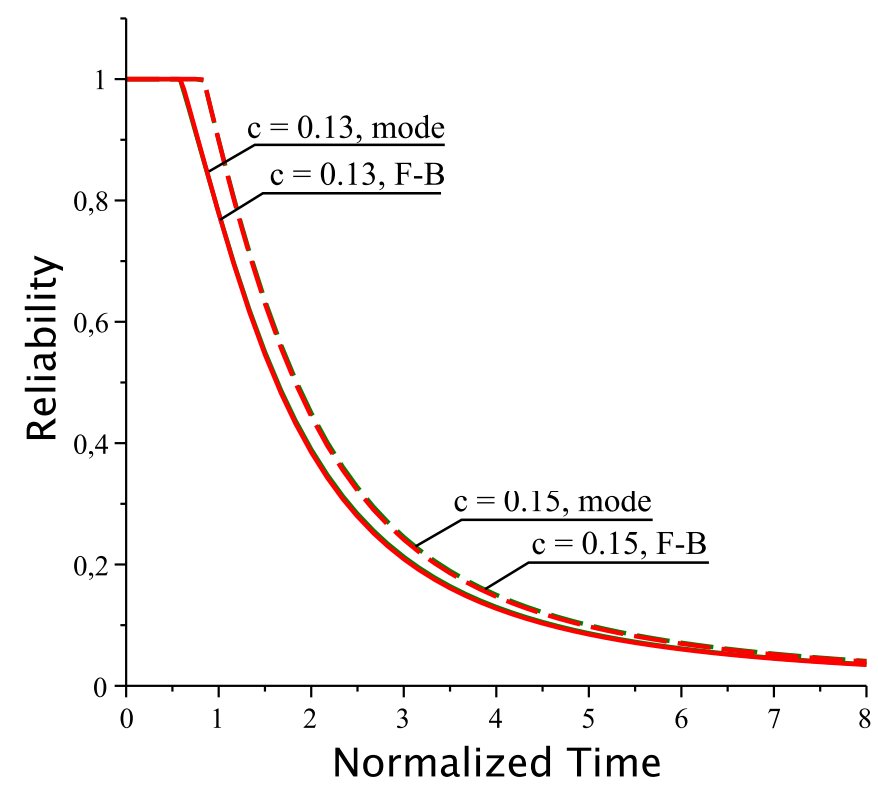

Fig. 5. Reliability as a function of time $(\alpha=1)$

and 6 show the reliability as a function of the first passagetime. The following values are adopted for the constants $\gamma=$ $2.27, \kappa=4.99$ and $\mu=1.588$. The solid curves relate to the case where $c=0.13$, whereas the dashed curves relate to the case where $c=0.15$.

It appears that the solution with the exact mode shape assigns a reliability that is not smaller than the one calculated from the approximation with the Filonenko-Borodich term. This holds for any applied load. For example, the reliability of a column under a loading of two times the critical load $(\alpha=2)$ and for the critical value $c=0.15$ gives $R(0.7)=0.904$ and $R^{*}(0.7)=0.892$, for $\alpha=1$ the following reliabilities are obtained $R(1)=0.90$ and $R^{*}(1)=0.90$ and for $\alpha=0.5$ we calculate $R(1.4)=0.94$ and $R^{*}(1.4)=0.93$.

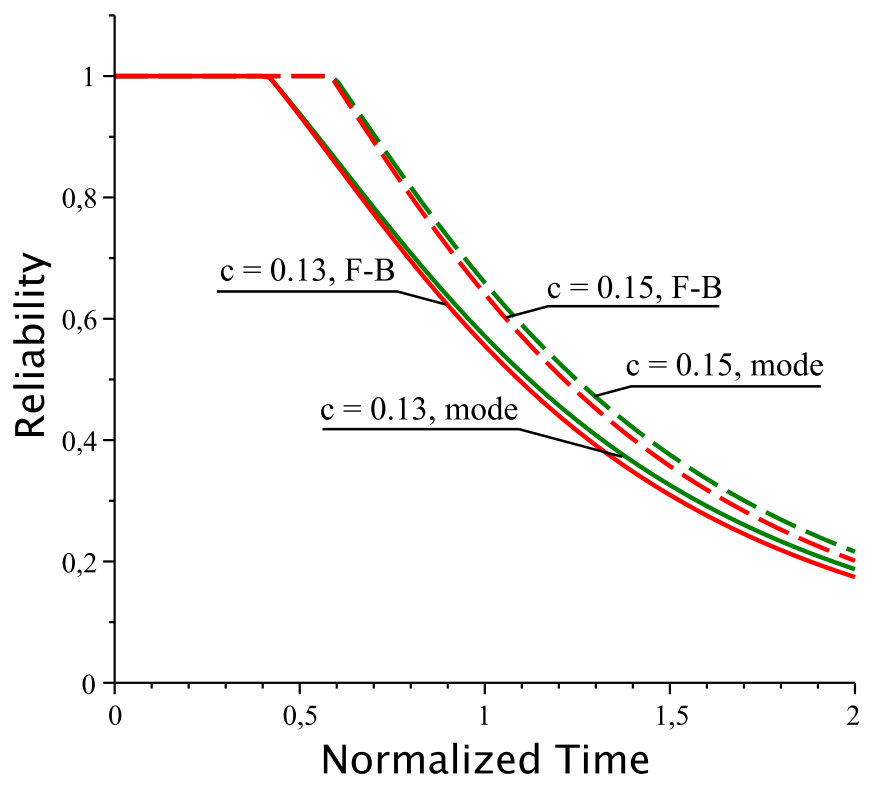

Fig. 6. Reliability as a function of time $(\alpha=2)$

This leads to the interesting conclusion that using the approximation will give us a conservative result.

\section{Comparison of Probabilistic and Interval Analyses}

The question arises how to compare the results from the probabilistic and interval analyses? The interval analysis provides the engineer with information on the upper bound of a quantity of interest related to the buckling problem given the upper bound on the interval variables that are present in the model. For example, the maximum possible deflection at any given point along the column and for any point in time is derived in section 4.1. The probabilistic analysis provides information on the reliability of the column given some failure criterion and the probability distribution of the random variables that are present in the model.

As the required reliability tends to unity, a clear relationship between the interval and probabilistic analyses is apparent. If failure in the probabilistic analysis is identified, as above, by the deflection surpassing a critical value $c$ and from the interval analysis it is clear that the deflection will not exceed this value $c$ up to a time $\tau_{0}$ for a given applied load, then the reliability is identical to unity up to this time $\tau_{0}$. This time frame can be considered the fail-safe life of the column.

Let us illustrate this with the examples already presented and concentrate on the solution with the exact mode shape and $\alpha=0.5$. The maximum attainable deflection of the midpoint of the column for the solution with the exact mode shape and $\alpha=0.5$ is depicted in figure 2 by the dashed green curve. The critical value $c=0.15$ is attained for $\tau_{0}=1.242$. In figure 4 the reliability defined as the probability that the mid-point of the column is not surpassing $c=0.15$ is depicted by the green dashed line for the solution with the exact mode shape. As the required reliability is approaching one, the values for the design life of the column $\tau$ of the 


\begin{tabular}{|r|c|} 
Reliability & Design Time $\tau$ \\
\hline 0.9 & 1.509 \\
0.95 & 1.373 \\
0.99 & 1.268 \\
0.995 & 1.255 \\
0.999 & 1.245 \\
0.9995 & 1.243 \\
1 & 1.242
\end{tabular}

Table 1. The design time for a clamped column under axial impact loading for different levels of the required reliability

probabilistic analysis converges to the fail-safe life $\tau_{0}$ as is clear from table 1 . Indeed, the reliability is equal to one till $\tau_{0}=1.242$.

Now that this relationship is made clear, the next, quite fundamental, question arises: which analysis is preferable? To this question, our answer should be balanced. If an engineer is only interested by the fail-safe life of a column, he does not need the entire probability distribution of the initial imperfection amplitude. The information on the fail-safe life is readily obtained by only having the bounds on the amplitude defined. However, if the engineer in the design phase would need more information apart from the binary outcome: fail or safe, he will have to resort to the probabilistic analysis. In such an analysis it is of utmost importance to have a correct and applicable probability distribution function of the initial imperfection amplitude available, to come up with reliable reliability calculations.

\section{Conclusions}

In this paper we investigate impact buckling of a column with initial imperfection. The latter is modeled in two ways: (1) probabilistic analysis, (2) interval analysis. It is shown that these two analyses converge to each other when the required reliability approaches unity. Numerical comparison is performed for the design time of the structure such that its reliability exceeds the required one.

\section{Achnowledgements}

This study was performed during the research stay of W.V. at the Florida Atlantic University. W.V. would like to acknowledge the highly appreciated support of the IWTFlanders, the Commission for Educational Exchange Between The United States of America, Belgium and Luxembourg (The Fulbright Fund and Vesuvius) and the Raymond Snoeys fund of the KU Leuven.

\section{References}

[1] Lindberg, H., 1965. "Impact buckling of a thin bar". ASME Journal of Applied Mechanics, 32, pp. 312-322.
[2] Elishakoff, I., 1978. "Axial impact buckling of a column with random initial imperfections". Journal of Applied Mechanics, 45(2), pp. 361-365.

[3] Elishakoff, I., 1978. "Impact buckling of thin bar via monte carlo method". Journal of Applied Mechanics, 45(3), pp. 586-590.

[4] Ben-Haim, Y., and Elishakoff, I., 1990. Convex Models of Uncertainty in Applied Mechanics. Elsevier.

[5] Qiu, Z., and Wang, X., 2006. "Interval analysis method and convex models for impulsive response of structures with uncertain-but-bounded external loads". Acta Mechanica Sinica, 22(3), pp. 265-276.

[6] Qiu, Z., Ma, L., and Wang, X., 2006. "Ellipsoidalbound convex model for the non-linear buckling of a column with uncertain initial imperfection". International Journal of Non-Linear Mechanics, 41(8), pp. 919-925.

[7] Hu, J., and Qiu, Z., 2010. "Non-probabilistic convex models and interval analysis method for dynamic response of a beam with bounded uncertainty". Applied Mathematical Modelling, 34(3), pp. 725-734.

[8] Lindberg, H., 1991. "Dynamic response and buckling failure measures for structures with bounded and random imperfections". Journal of Applied Mechanics, 58(4), pp. 1092-1095.

[9] Filonenko-Borodich, M., 1946. "On a certain system of functions and its applications in theory of elasticity". Prikladnaia Matematika i Mekhanika (Journal of Applied Mathematics and Mechanics), 10, pp. 193-208 (in Russian).

[10] Lindberg, H., and Florence, A., 1987. Dynamic pulse buckling: theory and experiment. Martinus Nijhoff Publishers, Dordrecht.

[11] Rao, S., 2007. Vibration of continuous systems. Wiley, New York.

[12] Vilenkin, N., 1952. "On some nearly periodic systems of functions". Prikladnaia Matematika i Mekhanika (Journal of Applied Mathematics and Mechanics), 16(3), pp. 812-814 (in Russian).

[13] Hoff, N., 1951. "The dynamics of the buckling of elastic columns". ASME Journal of Applied Mechanics, 18, pp. 68-71. 